\title{
Elaboração e avaliação de material educativo sobre a prevenção do câncer de mama
}

\author{
Elisa Kern de Castro* \\ Vanessa Teixeira ** \\ Michael Quadros Duarte ***
}

\section{Resumo}

Objetivo: Elaborar e avaliar a adequação de um material educativo para a prevenção do câncer de mama em mulheres. Método: O material educativo foi elaborado por pesquisadores da área de psicologia da saúde e sua avaliação foi feita por 34 profissionais da saúde (médicos, enfermeiras, nutricionistas). Utilizou-se um protocolo de avaliação do material educativo para a prevenção do câncer de mama. Os dados da avaliação foram analisados a partir de estatística descritiva e análise de conteúdo das respostas abertas. Resultados: Os profissionais da saúde avaliaram o material educativo de forma positiva, sugerindo pequenas alterações, como a exclusão do anticoncepcional como fator de risco, a inclusão do tabaco e uso de bebida alcoólica como fator de risco e mudanças nas características físicas das personagens. Conclusão: O material educativo está adequado ao público alvo e pode ser usado como material complementar em campanhas de educação em saúde para a prevenção do câncer de mama.

Palavras chave: Educação em saúde, Promoção da Saúde, Autocuidado, Câncer de mama.

\section{Evaluation Of Educational Material About The Prevention Of Breast Cancer}

\begin{abstract}
Purpose: To create and evaluate the adequacy of an educational material for the prevention of breast cancer in women. Method: The educational material was elaborated for health psychology researchers and its evaluation was done by 34 health professionals (physician, nurses, and nutritionists). A protocol was used to evaluate the educational material to breast cancer prevention. The data were analyzed using descriptive statistics and content analysis of open questions. Results: Health professionals evaluated the educational material in a positive way, suggesting small changes, such as exclusion of contraceptive as a risk factor, inclusion of tobacco and use of alcohol as a risk factor, and changes in the physical characteristics of the characters. Conclusion: The educational material is appropriate to the target audience and can be used as complementary material in health education campaigns for breast cancer prevention.
\end{abstract}

Key words: Health education, Health promotion, Self-care, Breast Cancer.

*Professora do Programa de Pós-Graduação em Psicologia da UNISINOS.

**Psicóloga pela UNISINOS.

*** Graduando em Psicologia na UNISINOS. 


\section{Introdução}

O câncer de mama (CM) é o tipo mais comum de câncer entre as mulheres do Brasil e do mundo, correspondendo a $25 \%$ dos novos casos da doença anualmente (Instituto Nacional do Câncer, 2016b). Pode também ser diagnosticado em homens, porém com uma estimativa baixa, cerca de 1\% do total de casos. O gênero (mulher), idade, herança genética, raça e etnia (branca) são fatores de risco para CM que não se pode alterar (American Cancer Society, 2015). Contudo, há outros fatores de risco passíveis de modificação e que dependem do comportamento da mulher, como o consumo de álcool excessivo, estar acima do peso ou casos de obesidade após a menopausa, nuliparidade, ter filhos após os trinta anos, uso de contraceptivos orais e injetáveis e uso de terapia hormonal após a menopausa. Como fatores de proteção para o CM, estão as atividades físicas e amamentação. (American Cancer Society, 2015). A grande maioria dos fatores de riscos conhecidos pelas mulheres são do tipo modificáveis (comportamentos e estilo de vida), o que pode favorecer a adoção de práticas preventivas (Batiston, Tamaki, Souza \& Santos, 2011).

Existem alguns sinais e sintomas que podem ser considerados indícios de alteração nas mamas, entre eles o aparecimento de nódulos; pele da mama avermelhada, retraída ou com uma aparência de casca de laranja; alterações nos mamilos; pequenos nódulos nas axilas ou pescoço e saída de líquido anormal nas mamas. (Instituto Nacional do Câncer, 2016a). A mamografia, a ecografia mamária e o exame clínico nas mamas são os exames existentes para prevenção e/ou diagnóstico precoce do CM (Feldmann, 2015). Segundo a Lei 11.664 de 29 de abril de 2008 (Brasil, 2008), no Brasil, toda a mulher acima de 40 anos de idade tem o direito de realizar o exame de mamografia de caráter preventivo. A realização da mamografia é recomendada em mulheres com idade entre 50 e 69 anos, com um intervalo de dois anos (Instituto Nacional do Câncer, 2016a). Os exames de rastreamento não excluem a realização do autoexame mamário (AEM), que tem o objetivo de auxiliar a mulher a ter mais conhecimento sobre seu corpo, podendo detectar precocemente qualquer tipo de anormalidade em suas mamas (Santana, 2015). Porém, o modo como realizá-lo é ainda pouco conhecido entre a população feminina, reforçando a necessidade de campanhas de educação em saúde para promover o autocuidado (Silva, Lício, Borges, Mendes, Vicente \& Gomes, 2012; Seabra, 2016).

$O$ conceito de autocuidado refere-se às ações que visam o bem-estar físico e emocional referente a compor- tamentos do cotidiano, realizados pelo próprio indivíduo para a manutenção da sua saúde (Castro \& Moro, 2012). Um dos princípios fundamentais do autocuidado refere-se ao fato de que qualquer indivíduo consciente possui o direito de realizar escolhas que o responsabilize com os cuidados voltados ao seu corpo, visando um maior conforto e satisfação. (Lunardi, Lunardi Filho, Silveira, Soares \& Lipinski, 2004). O autocuidado que o indivíduo tem da sua saúde é influenciado por percepções ou crenças individuais sobre a doença, que são dinâmicas, construídas e reconstruídas ao longo de sua vida (Castro, Peuker, Lawrenz \& Figueiras, 2015; Leventhal, Bodnar-Deren, Breland, Hash-Converse, Phillips, Leventhal \& Cameron, 2012).

Em Psicologia da Saúde, o modelo teórico do Senso Comum (MSC) (Leventhal, Brissette \& Leventhal, 2003) parte do pressuposto de que os indivíduos elaboram individualmente as suas crenças em saúde a partir dos seus próprios esquemas mentais. Essas crenças podem ser compreendidas a partir das dimensões: 1 ) identidade (sintomas da doença); 2) duração (aguda, crônica ou cíclica); 3) causas; 4) consequências (que tipo de sequela ou desfecho a doença pode gerar); 5) controle e/ou cura (crença de controle pessoal ou do tratamento)(Leventhal, Brissette \& Leventhal, 2003); 6) coerência (compreensão individual da doença); 7) representação emocional (Moss-Moris, Weinman, Petrie, Horne, Cameron \& Buick, 2002). Uma revisão sistemática (Seabra, Peuker \& Castro,2015) que compilou as contribuições do MSC em pesquisas sobre o CM, constatou-se que as crenças desajustadas da doença podem impactar em alterações psicológicas, gerando ansiedade e depressão, e dificultar os comportamentos em saúde. Na pesquisa que norteou o desenvolvimento do presente material educativo (Seabra, 2015), observou-se que as mulheres saudáveis podem confundir os efeitos colaterais ocasionados pelo tratamento contra o $\mathrm{CM}$ (quimioterapia e radioterapia) com os sintomas da doença. Referiram o CM como ameaçador e com consequências graves à sua saúde, e com causas emocionais e comportamentais.

Assim, apenas informação não é suficiente para mudar comportamento em saúde (Castro et al., 2015; Peuker, Armiliato, Souza \& Castro, 2016), sendo necessário criar estratégias de educação em saúde baseado não só nas informações concretas da doença, mas nas crenças das pessoas a respeito delas (Lima, Peuker \& Castro, 2016; Castro et al., 2015). Os materiais elaborados e destinados a ações de promoção em saúde devem abranger sempre estratégias de educação, incluindo conteúdos que propi- 
ciam a adesão dos pacientes a determinado tratamento e/ ou à prevenção de comportamento que levem aos fatores de risco de uma determinada doença (Gorayeb \& Nakao, 2015). Para isso, esses materiais devem ser elaborados dentro do contexto de cada público alvo, com escrita de fácil compreensão e adequada com a cultura. Os materiais impressos desenvolvidos na área de educação em saúde têm tripla função: 1) reforçar as orientações verbalizadas; 2) ser um guia para dúvidas posteriores; 3) auxiliar nas tomadas de decisões (Lima, Peuker \& Castro, 2016; Moreira, Nóbrega \& Silva, 2003).

Considerando o que foi exposto, o presente artigo visa apresentar a elaboração de um material educativo para a prevenção do câncer de mama a partir de uma pesquisa que avaliou as crenças de mulheres saudáveis sobre a doença (Seabra, 2015) e avaliar a sua adequação a partir da visão técnica de profissionais da saúde.

\section{MÉTODO:}

\section{Delineamento: Descritivo, transversal.}

Participantes: A avaliação do material foi feita por 34 profissionais da área da saúde atuantes em saúde da mulher em Unidades Básicas de Saúde (UBS) ou hospitais públicos e privados. Dentre eles, dez eram médicos $(29,4 \%), 22$ enfermeiros $(64,7 \%)$ e dois nutricionistas $(5,9 \%)$. Os participantes possuíam em média 12,76 anos de formação superior (DP = 10,67) e 10,09 anos de experiência profissional ( $\mathrm{DP}=6,92)$. Os critérios de inclusão no estudo para a amostra de profissionais foram: formação de nível superior e atuação no campo da saúde; ter tido alguma experiência profissional na área de Atenção Básica e/ou experiência em educação em saúde (atuação na atenção básica com projetos de prevenção, promoção da saúde e elaboração de tecnologias para a saúde).

Coleta de dados: Os locais para a coleta de dados foram Unidades Básicas de Saúde (UBS) e dois hospitais (um público e um privado/convênios) em Porto Alegre e região metropolitana. O convite para a participação dessas instituições se deu mediante explicação da pesquisa às chefias, que indicaram os profissionais para participação na pesquisa. Após o preenchimento do Termo de Consentimento Livre e Esclarecido (TCLE), os profissionais receberam um envelope contendo o material educativo proposto (entregue em uma via impressa em cores) e o instrumento de avaliação do mesmo. Eles ficaram de posse do envelope por alguns dias, e, após, a pesquisadora recolheu todos os questionários dos profissionais daquele local, preenchidos de forma anônima.

Material educativo: Foi baseado nos resultados da pesquisa de Seabra (2015), realizada com 211 mulheres atendidas em uma UBS que avaliou a percepção de risco, a percepção da doença e o autocuidado de mulheres saudáveis em relação ao CM. Os resultados do estudo apontaram que as participantes apresentaram baixa compreensão sobre os fatores que envolvem o CM, apesar de acreditarem que têm conhecimento sobre a doença (Seabra, 2015). O conteúdo da cartilha, visando a prevenção do câncer de mama, foi elaborado por pesquisadores com experiência em psicologia da saúde, no formato de uma história em quadrinhos, em que se buscou explorar a ideia de personagens que contemplassem mulheres em diferentes faixas etárias e diferentes preocupações com sua saúde (material em anexo).

Instrumentos: Foi elaborado um instrumento para avaliação do material, baseado no estudo de Lima, Peuker e Castro (2016), que seguiu orientações de Doak, Doak \& Root (1996), os quais utilizaram um método denominado Suitability Assessment of Materials (SAM). Nesse método, deve constar no protocolo de avaliação, seis áreas a serem avaliadas: (1) conteúdo do material educativo; (2) adequação da linguagem; (3) gráficos; (4) layout; (5) estímulo da aprendizagem; e (6) adequação cultural. O instrumento foi dividido em três partes: a) Parte 1: Caracterização da experiência profissional da amostra; b)Parte 2: Apresentação do enredo e os personagens da história. Caso o especialista considerasse incorreta ou inadequada alguma das informações contidas no material, era solicitado que apontasse qual a correção a ser feita e alternativas para sua melhoria; c) Parte 3: Questões sobre o conteúdo da cartilha como, por exemplo, o estímulo à aprendizagem e motivação e a linguagem utilizada no material e em relação aos materiais educativos em saúde, visando uma avaliação do material como um todo. $\mathrm{O}$ instrumento contemplou perguntas relativas à avaliação do conteúdo sob o ponto de vista técnico, bem como da adequação da linguagem ao público alvo (ex: se a linguagem é clara, se os conceitos são abordados de formas objetivas).

Procedimentos éticos: O projeto maior, no qual foi baseado para a construção do material educativo, foi aprovado pelo comitê de Ética em Pesquisa da Universidade do Vale do Rio dos Sinos (180/2014). 
Procedimentos para análise de dados: Os dados coletados foram tabulados no programa SPSS versão 22.0. Foram realizadas análises descritivas dos resultados para a verificação das médias, desvio-padrão, frequências e percentagem das respostas. Foi realizada análise de conteúdo das respostas abertas em que haviam sugestões de aprimoramento do material.

\section{RESULTADOS:}

Todos os profissionais avaliaram que o material educativo apresentado pode contribuir na prevenção do câncer de mama. Em relação à sua adequação com o público alvo, todos eles também consideraram que as informações estavam apropriadas, e 94,1\% dos participantes $(n=32)$ avaliaram que a linguagem utilizada é compreensível e clara à população destinada, conforme fala de um profissional: "Material de linguagem fácil, que o público consegue entender".

Trinta e dois profissionais $(97,0 \%)$ afirmaram que conceitos importantes sobre o CM são apresentados de forma clara e objetiva. Os termos e palavras utilizados nos textos foram avaliados de fácil compreensão por 32 $(97,0 \%)$ profissionais. “Acho o material com uma linguagem adequada e acessível". (P10).Os dois profissionais que avaliaram que a linguagem do material não estava clara à população destinada sugeriram a revisão ortográfica do material, sem, entretanto, sugerir alterações em falas específicas. A falta de informações no material educativo sobre o CM foi apontada por sete $(21,9 \%)$ profissionais. Desses profissionais, dois deles sugeriram a inclusão do uso de tabaco como fator de risco e dois deles a inclusão da ilustração do modo como deve se realizar o autoexame. Um profissional sugeriu a inclusão da informação dos alimentos que podem contribuir para o desenvolvimento da doença, e outro, sobre a dor na realização da mamografia, apontando que o incômodo pode variar para cada mulher. "Acho que referente a dor da mamografia é muito relativo, pode ser colocado que varia de mulher o incômodo"(P30).

Vinte e oito profissionais $(90,3 \%)$ avaliaram que é de muita importância a elaboração de materiais educativos em conjunto com profissionais de diferentes áreas disciplinares. A partir dos apontamentos dos profissionais, o material educativo sofreu algumas modificações, sendo então que o material final foi elaborado levando em conta os ajustes e sugestões dos profissionais de saúde.

O quadro 1 compila as informações do material educativo e apresenta as falas originais das personagens na primeira versão do material, as falas modificadas após avaliação do mesmo, e as crenças sobre o câncer de mama trabalhadas em cada uma delas.

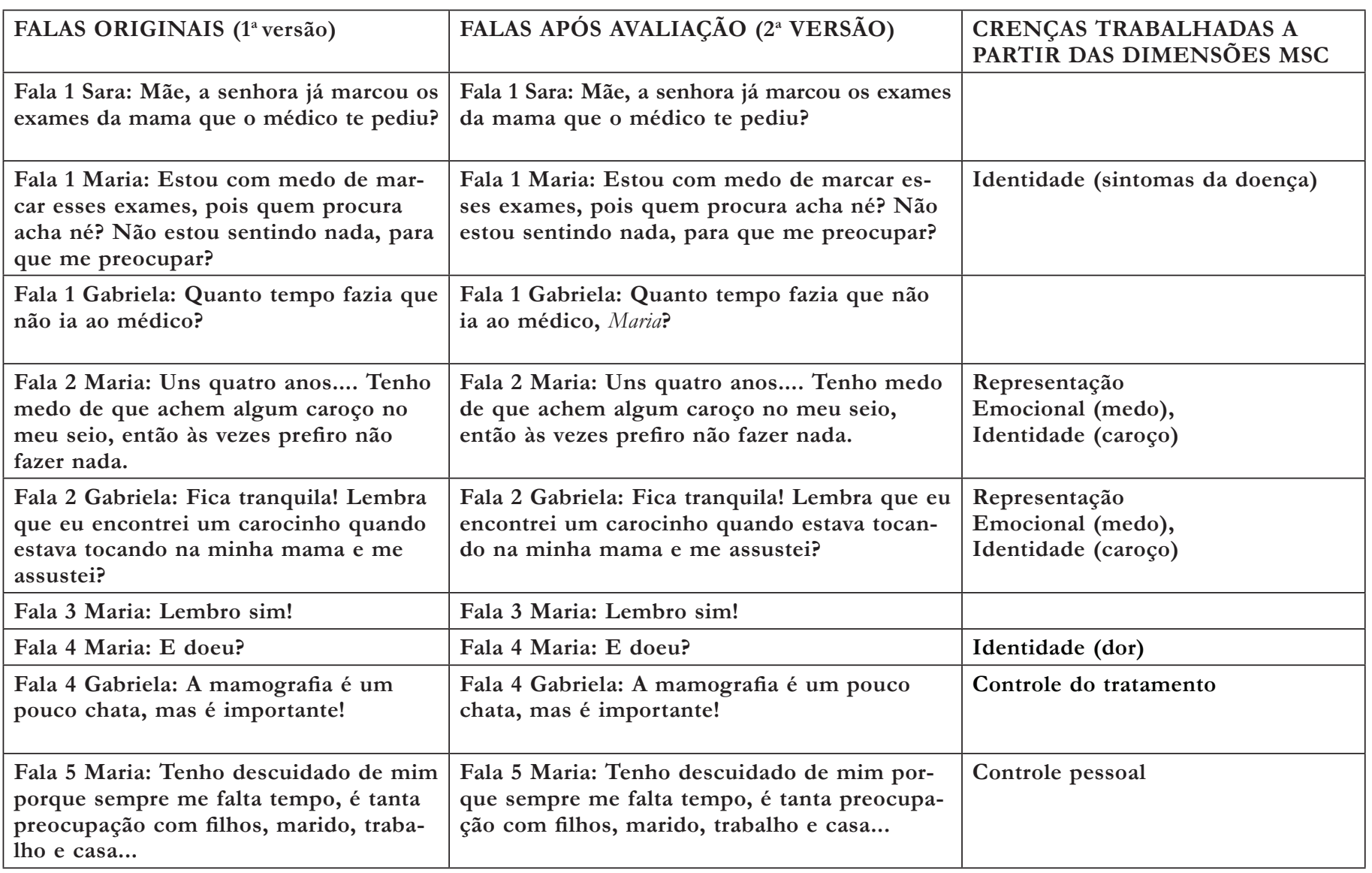


Fala 2 Sara: Acho melhor marcar os exames então mãe! Gabriela, você sabe quem tem mais chance de ter câncer de mama?

Fala 5 Gabriela: Existem vários fatores, como ter casos na família, ter mais de 40 anos, usar hormônios por muito tempo como a pílula anticoncepcional, e outros...

Fala 3 Sara:Nossa! Quanta coisa, pensei que fosse só de família! $\mathrm{E}$ o que podemos fazer para não ter câncer?

Fala 6 Gabriela: Você está realizando um neste momento Sara, que é a amamentação.

Fala 6 Maria: E o que mais, Gabriela?

Fala 7 Gabriela:Cuidar da saúde todos os dias, fazer algum esporte, comer bem, como frutas e verduras, é muito importante!

Fala 7 Maria: Tenho que cuidar da minha saúde!

Fala 8 Gabriela: Foi cuidando de minha saúde que descobri o caroço na mama, que ainda bem não era câncer, mas poderia ter sido!

Fala 4 Sara: Que bom que não era câncer!

Fala 9 Gabriela: Mas, mesmo que fosse câncer, hoje em dia existem tratamentos muito bons, ainda mais se descobrimos no início da doença.

Fala 5 Sara: A partir de qual idade são indicados esses exames que você fez Gabriela?

Fala 10 Gabriela: Estes exames que eu fiz são indicados a partir dos quarenta anos, mas as mulheres de qualquer idade podem se examinar sozinhas e, se encontrarem algum carocinho, ir ao médico.

Fala 6 Sara: Quantas dúvidas você me esclareceu, vou continuar amamentando, fazer o autoexame nas mamas e ir ao médico com frequência, para continuar cuidando de mim. Mãe, vamos nos cuidar!

Fala 8 Maria: Vou marcar os exames amanhã mesmo!

Frase final: E você está ou já esteve na mesma situação da Maria? A Maria descobriu que não tinha nada, mas caso tivesse descoberto algo, existem tratamentos para cada caso e estagio da doença. Cuide-se!
Fala 2 Sara: Acho melhor marcar os exames então mãe! Gabriela, você sabe quem tem mais chance de ter câncer de mama?

Fala 5 Gabriela: Existem vários fatores, como ter casos na família, principalmente se for mãe ou irmã, ter mais de 40 anos, com maior chance de desenvolver após os 50 anos de idade, usar hormônios por muito tempo, e outros...

Fala 3 Sara:Nossa! Quanta coisa, pensei que fosse só de família! E o que podemos fazer evitar o câncer?

Fala 6 Gabriela: Você está realizando um neste momento Sara, que é a amamentação.

Fala 6 Maria: E o que mais, Gabriela?

Fala 7 Gabriela:Cuidar da saúde todos os dias, faça algum exercício físico, coma bem incluindo frutas e verduras na sua dieta, evite fumar e usar bebidas alcóolicas.

Fala 7 Maria: Tenho que cuidar da minha saúde!

Fala 8 Gabriela: Foi cuidando de minha saúde que descobri o caroço na mama, que ainda bem não era câncer, mas poderia ter sido!

Fala 4 Sara: Que bom que não era câncer!

Fala 9 Gabriela:Mas, mesmo se fosse câncer, hoje em dia existem tratamentos muito bons, ainda mais se descobrimos no início da doença.

Fala 5 Sara: A partir de qual idade são indicados esses exames que você fez Gabriela?

Fala 10 Gabriela: São indicados a partir dos quarenta anos, mas as mulheres de qualquer idade podem se examinar sozinhas e, se encontrarem algum carocinho, ir ao médico.

Fala 6 Sara:Quantas dúvidas você me esclareceu! Vou continuar amamentando, fazer o autoexame nas mamas e ir ao médico com frequência, fazendo os exames de rotina.

s
(

\begin{tabular}{|c|c|}
\hline $\begin{array}{l}\text { Fala } 2 \text { Sara: Acho melhor marcar os exames } \\
\text { então mãe! Gabriela, você sabe quem tem } \\
\text { mais chance de ter câncer de mama? }\end{array}$ & Controle pessoal e do tratame \\
\hline $\begin{array}{l}\text { Fala } 5 \text { Gabriela: Existem vários fatores, como } \\
\text { ter casos na família, principalmente se for mãe } \\
\text { ou irmã, ter mais de } 40 \text { anos, com maior chan- } \\
\text { ce de desenvolver após os } 50 \text { anos de idade, } \\
\text { usar hormônios por muito tempo, e outros... }\end{array}$ & Causas (fatores de risco) \\
\hline $\begin{array}{l}\text { Fala } 3 \text { Sara:Nossa! Quanta coisa, pensei que } \\
\text { fosse só de família! E o que podemos fazer } \\
\text { evitar o câncer? }\end{array}$ & $\begin{array}{l}\text { Causa da doença, } \\
\text { Controle pessoal }\end{array}$ \\
\hline $\begin{array}{l}\text { Fala } 6 \text { Gabriela: Você está realizando um neste } \\
\text { momento Sara, que é a amamentação. }\end{array}$ & Controle pessoal \\
\hline Fala 6 Maria: E o que mais, Gabriela? & Controle pessoal \\
\hline $\begin{array}{l}\text { Fala } 7 \text { Gabriela: Cuidar da saúde todos os dias, faça } \\
\text { algum exercício físico, coma bem incluindo frutas e verdu- } \\
\text { ras na sua dieta, evite fumar e usar bebidas alcóolicas. }\end{array}$ & Controle pessoal \\
\hline $\begin{array}{l}\text { Fala } 7 \text { Maria: Tenho que cuidar da minha } \\
\text { saúde! }\end{array}$ & Controle pessoal \\
\hline $\begin{array}{l}\text { Fala } 8 \text { Gabriela: Foi cuidando de minha saúde } \\
\text { que descobri o caroço na mama, que ainda } \\
\text { bem não era câncer, mas poderia ter sido! }\end{array}$ & $\begin{array}{l}\text { Identidade (caroço), } \\
\text { Consequência (não era câncer }\end{array}$ \\
\hline Fala 4 Sara: Que bom que não era câncer! & \\
\hline $\begin{array}{l}\text { Fala } 9 \text { Gabriela:Mas, mesmo se fosse câncer, hoje em } \\
\text { dia existem tratamentos muito bons, ainda mais se desco- } \\
\text { brimos no início da doença. }\end{array}$ & $\begin{array}{l}\text { Controle do tratamento, conse } \\
\text { cias (detecção precoce e chan } \\
\text { cura) }\end{array}$ \\
\hline $\begin{array}{l}\text { Fala } 5 \text { Sara: A partir de qual idade são indica- } \\
\text { dos esses exames que você fez Gabriela? }\end{array}$ & Controle pessoal e do tratame \\
\hline $\begin{array}{l}\text { Fala } 10 \text { Gabriela: São indicados a partir dos } \\
\text { quarenta anos, mas as mulheres de qualquer } \\
\text { idade podem se examinar sozinhas e, se en- } \\
\text { contrarem algum carocinho, ir ao médico. }\end{array}$ & $\begin{array}{l}\text { Controle pessoal, Identidade, } \\
\text { ração }\end{array}$ \\
\hline $\begin{array}{l}\text { Fala } 6 \text { Sara:Quantas dúvidas você me esclareceu! Vou } \\
\text { continuar amamentando, fazer o autoexame nas mamas e } \\
\text { ir ao médico com frequência, fazendo os exames de rotina. }\end{array}$ & $\begin{array}{l}\text { Causa, Controle pessoal e do } \\
\text { mento }\end{array}$ \\
\hline
\end{tabular}

Fala 8 Maria: Vou marcar os exames amanhã mesmo!

Frase final: $E$ você está ou já esteve na mesma situação da Maria? A Maria descobriu que não tinha nada, mas caso tivesse descoberto algo, existem tratamentos para cada caso e estagio da doença. Cuide-se!

Inclusão da ilustração da ecografia mamária e alteração nas características físicas das personagens.

Controle do tratamento

Consequência, controle pessoal e do tratamento, duração (doença crônica/aguda) 


\section{Discussão}

A partir dos resultados percebe-se que o material educativo foi avaliado positivamente pelos profissionais, com algumas sugestões de alterações no conteúdo da história, ajustes que são comuns quando se avalia esse tipo de material (Lima, Peuker \& Castro, 2016). Os profissionais, em sua maioria, apontaram que a linguagem utilizada no material é de fácil compreensão.

$\mathrm{Na}$ construção das falas das personagens, objetivou-se trabalhar as crenças sobre o câncer de mama que o estudo de Seabra (2015) identificou como importantes de serem abordadas. A elaboração do material educativo baseado num modelo teórico claro - Modelo do Senso Comum - (Leventhal, Brissette \& Leventhal, 2003) e com fortes evidências empíricas do contexto brasileiro (Seabra, 2015) possibilitaram a criação de uma história em que se aproxima o saber popular e o científico. Além disso, ao aproximar-se da cultura das usuárias, o texto torna-se de fácil compreensão e podem ser utilizadas frases comuns ao cotidiano das mulheres, atingindo o público que se destina (DeWalt, Callahan, Hawk, Broucksou, Hink, Rudd \& Brach, 2010; Echer, 2005). Tenta-se informar e desfazer percepções distorcidas sobre a realização da mamografia, bem como sobre o câncer e os tratamentos indicados (Assis \& Mamede, 2016; Seabra, 2015).

O material educativo pode auxiliar as mulheres na compreensão da doença e na comunicação da equipe de saúde com as mesmas, facilitando o entendimento sobre os meios de prevenção e fatores de risco, de forma clara e simples (Lima, Peuker \& Castro, 2016). Esse material está voltado para a área da educação em saúde, cujo o objetivo é o de modificar saberes já existentes, visando a ampliação do conhecimento, tornando o indivíduo capaz de cuidar de si a partir de sua autonomia (Souza \& Jacobina, 2009). Um diferencial do presente material é estar baseado em conhecimento científico (Seabra, 2015), buscando a compreensão das mulheres na assimilação de novos comportamentos em saúde.

Cabe ressaltar que a informação como modo de educação em saúde tem como estratégia fazer despertar a autonomia do indivíduo na busca por meios de prevenção da doença. Os materiais educativos sobre o $\mathrm{CM}$ objetivam uma maior visibilidade da doença às mulheres, esclarecendo dúvidas e desmistificando a doença em si. (Moreira, Bernardo \& Catunda, 2013). O material elaborado neste estudo, a partir das alterações sugeridas pelos profissionais, objetiva estimular nas mulheres o autocuidado, bem como mudanças de comportamento, fomentando a prevenção.

\section{Conclusão}

Este estudo possibilitou a elaboração e a avaliação da adequação do material educativo para prevenção do câncer de mama baseado em evidência científica da área da psicologia da saúde. O material parece estar adequado a partir do ponto de vista dos profissionais, e foi aprimorado a partir dos resultados desse estudo. É importante salientar que, diferente de outros materiais educativos em saúde existentes, esse material foi baseado em modelos teóricos sólidos de psicologia da saúde e de conhecimentos gerados a partir da visão de pessoas que estão inseridas na realidade que o material será distribuído. Nesse sentido, a grande contribuição do material educativo, além de usar uma história em quadrinhos de forma lúdica, busca trabalhar as crenças mais frequentes nas mulheres saudáveis frente ao câncer de mama, expondo também informações importantes para sua prevenção. Possíveis limitações do presente material educativo dizem respeito à dificuldade de atingir pessoas de pouca escolaridade e / ou com dificuldades de leitura e interpretação de texto. Contudo, essa dificuldade é encontrada frente a qualquer material impresso que requeira um nível de leitura mínimo, e, para atingir pessoas menos escolarizadas, será preciso lançar mão de outras técnicas e materiais que possam ajudá-las a conhecer o câncer de mama e se prevenir.

\section{Referências bibliográficas}

American Cancer Society (2015). Breast Cancer Prevention and Early Detection. Atlanta, 2015.

Assis, C. F., \& Mamede, M. (2016). A Mamografia e seus Desafios: Fatores Socioeducacionais Associados ao Diagnóstico Tardio do Câncer de Mama. Iniciação Científica Cesumar, 18(1), 63-72.

Batiston, A. P., Tamaki, E. M., Souza, L. A. D., \& Santos, M. L. D. M. D. (2011). Conhecimento e prática sobre os fatores de risco para o câncer de mama entre mulheres de 40 a 69 anos. Revista Brasileira Saúde Materno Infantil, Recife, v. 11, n. 2, p. 163-171, abr. /jun. 2011.

Brasil (2008). Decreto Lei $\mathrm{n}^{\circ} 11.664$, de 29 de abril de 2008. Dispõe sobre a efetivação de ações de saúde que assegurem a prevenção, a detecção, o tratamento e o seguimento dos cânceres do colo uterino e de mama, no âmbito do Sistema Único de Saúde - SUS. Brasília, 2008a.

Castro, E. K., Moro, L. (2012). Factores psicosociales relacionados con el autocuidado en la prevención, tratamiento y postratamiento del cáncer de mama. Psicooncología, 9, (2-3), 453-465.

Castro, E. K. D., Peuker, A. C., Lawrenz, P. \& Figueiras, M. J. (2015). Illness perception, knowledge and self-care about cervical cancer. Psicologia: Reflexão e Crítica, 28(3), 483-489.

DeWalt, D., Callahan, L., Hawk, V., Broucksou, K., Hink, A., Rudd, R. \& Brach, C. (2010). Health Literacy Universal Precautions Toolkit.(Prepared by North Carolina Network Consortium, The Cecil G. Sheps Center for Health Services Research, The University of North Carolina at Chapel Hill, under Contract No. HHSA290200710014. AHRQ Publication No. 10-0046-EF). Rockville, MD: Agency for Healthcare Research and Quality.

Doak, C. C., Doak, L. G. \& Root, J. H. (1996). Teaching patients with low literacy skills. AJN The American Journal of Nursing, 96 (12), 16.

Echer, I. C. (2005). Elaboração de manuais de orientação para o cuidado em saúde. Revista Latino-Americana de Enfermagem, 13(5), 754-757.

Feldmann, A. F. (2015). Comunicação, gênero e saúde uma análise das campanhas do câncer de mama no Brasil. São Paulo: Atlas. 
Gorayeb, R. \& Nakao, R. (2015). Educação em Saúde. In: Gorayeb (org). A prática da Psicologia no Ambiente Hospitalar. Novo Hamburgo: Sinopsys. Instituto Nacional do Câncer (2016b). Tipos de câncer. Rio de Janeiro, 2016.

Instituto Nacional do Câncer (2016a). Detecção precoce para o câncer de mama. Rio de Janeiro, 2016.

Leventhal, H., Brissette, I. \& Leventhal, E. A. (2003). The common-sense model of self-regulation of health and illness. The self-regulation of health and illness behaviour, 1, 42-65.

Leventhal, H., Bodnar-Deren, S., Breland, J. Y., Hash-Converse, J., Phillips, L. A., Leventhal, E. A., \& Cameron, L. D. (2012). Modeling Health and Illness Behavior. In J. Bawn, A., Revenson, T. A. \& Singer (Ed.), Handbook of Health Psychology (pp. 3-35). New York: Routledge Handbooks Online.

Lima, N. B., Peuker, A. C. W. \& Castro, E. K.(2016). Avaliação de material educativo sobre a prevenção do câncer de colo de útero. Revista Sul-Brasileira de Enfermagem, 22(1), 5-13.

Lunardi, V. L., Lunardi Filho, W. D., da Silveira, R. S., Soares, N. V. \& Lipinski, J. M. (2004). O cuidado de si como condição para o cuidado dos outros na prática de saúde. Revista Latino-Americana de Enfermagem, 12(6), 933-939.

Moreira, C. B., Bernardo, E. B. R., \& Catunda, H. L. O. (2013). Construção de um vídeo educativo sobre detecção precoce do câncer de mama. Revista Brasileira de Cancerologia, 59(3), 401-407.

Moreira, M. D. F., Nóbrega, M. M. L. \& Silva, M. I. T. (2003). Comunicação escrita: contribuição para a elaboração de material educativo em saúde. Revista Brasileira de Enfermagem, 56(2), 184-188.
Moss-Morris, R., Weinman, J., Petrie, K., Horne, R., Cameron, L., \& Buick, D. (2002). The revised illness perception questionnaire (IPQ-R). Psychology and health, 17(1), 1-16.

Peuker, A. C. W., Armiliato, M. J., Souza, L. V. D. \& Castro, E. K. D. (2016). Causal attribution among women with breast cancer. Psicologia: Reflexão e Critica, 29.

Santana, L. F. (2015). O autoexame de mama como método preventivo para o câncer mamário. Caderno Discente, 2(1).

Seabra, C. R., Peuker, A. C. W. B., \& de Castro, E. K. (2015). Modelo de Autorregulação em Saúde e câncer de mama: uma revisão sistemática de literatura. Saúde e Desenvolvimento Humano, 3(2), 79-105.

Seabra, C. (2016). Percepção da doença e de risco e autocuidado para a detecção precoce do câncer de mama no contexto da saúde pública. Dissertação Mestrado, Universidade do Vale do Rio dos Sinos (UNISINOS), São Leopoldo, 2016.

Silva, S. R., Lício, F. C., Borges, L. V., Mendes, L. C., Vicente, N. G., \& Gomes, N. S. (2012). Atividades educativas na área da saúde da mulher: um relato de experiência. Revista de Enfermagem e Atenção à Saúde, 1(1), 106-112.

Souza, I. P. M. A. D. \& Jacobina, R. R. (2009). Educação em saúde e suas versões na história brasileira. Rev. baiana saúde pública, 33(4).

Submetido em: 3-4-2017

Aceito em: 3-10-2017 